\title{
SHORT TIME ASYMPTOTICS OF RANDOM HEAT KERNELS
}

\author{
Hiroshi KUNITA \\ Graduate School of Mahtematics \\ Kyushu University 36 \\ Fukuoka 812-8581, Japan \\ kunita@math.kyushu-u.ac.jp
}

\begin{abstract}
We study the random fundamental solution of a linear stochastic parabolic partial differential equation of the second order. We are particularly interested in the short time asymptotics of the random fundamental solution. If the random differential operator is nondegenerate, it will be shown that the short time asymptotics is represented by a Gaussian kernel. However, if it is degenerate, the short time aymptotics will be represented by a joint distribution of a certain linear sum of a Brownian motion and its multiple Wiener-Stratonovich integrals.

Our approach is based on the asymptotic analysis of the stochastic flow which solves the stochastic partial differential equation. It is parallel to the study of the short time asymptotics of the fundamental solution of a second order (deterministic) partial differerntial equation in Kunita [3].
\end{abstract}

\section{SHORT TIME ASYMPTOTICS OF HEAT KERNELS}

Let us consider a second order linear partial differential operator $L$ on a Euclidean space $\mathbf{R}^{d}$ represented by

$$
L=\frac{1}{2} \sum_{j=1}^{r} X_{j}^{2}+X_{0},
$$

where $X_{0}, X_{1}, \ldots, X_{r}$ are first order linear partial differential operators (=vector fields) with $C^{\infty}$ coefficients. We assume that $X_{0}, X_{1}, \ldots, X_{r}$ are complete and generate an $n$ dimensional Lie algebra $\mathcal{L}$, where $d \leq n<\infty$. In [3], the author studied the short time asymptotics of the fundamental solution associated with the operator $L$, through the solution of a stochastic differential equation:

$$
d \varphi_{t}=\sum_{j=1}^{r} X_{j}\left(\varphi_{t}\right) \circ d W^{j}(t)+X_{0}\left(\varphi_{t}\right) d t
$$

The original version of this chapter was revised: The copyright line was incorrect. This has been corrected. The Erratum to this chapter is available at DOI: 10.1007/978-0-387-35359-3_40 
where $\left(W^{1}(t), \ldots, W^{r}(t)\right)$ is a standard $r$-dimensional Brownian motion on the probability space $(\Omega, \mathcal{F}, P)$ and $\circ d W^{j}(t)$ are Stratonovich integrators. We shall summarize them in a different context. In the following discussions, Condition (A.2) in [3] will be not assumed, but results will be stated in a localized form.

We first consider the case where $L$ is nondegenerate. Let $\varphi_{t}(x), t \geq 0$ be the solution of equation (2) starting from $x$ at time 0 . It is a diffusion process with the infinitesimal generator $L$. Let $P_{t}(x, E)$ be the distribution of the random variable $\varphi_{t}(x)$. It is not a Gaussian distribution unless $X_{j}, j=1, \ldots, r$ are constant vectors (first order differential operators with constant coefficients). However, if $X_{1}, \ldots, X_{r}$ are commutative and $X_{0}=\sum_{j=1}^{r} c^{j} X_{j}$, then we can choose a local coordinate system $\left\{\left(\psi_{x}, U_{x}\right), x \in \mathbf{R}^{d}\right\}$ such that for any $x \in \mathbf{R}^{d}$, the distribution $P_{t}(x, E)$ on the local coordinate $\left(\psi_{x}, U_{x}\right)$ is Gaussian.

We will show this in the case where $r=d$ and $X_{1}, \ldots, X_{d}$ are linearly independent. Associated with $\left\{X_{1}, \ldots, X_{d}\right\}$ we consider a system of maps $\phi_{x} ; \mathbf{R}^{d} \rightarrow \mathbf{R}^{d}$ with parameter $x$ by

$$
\phi_{x}(z)=\phi_{x}\left(z_{1}, \ldots, z_{d}\right):=\exp \left\{\sum_{j=1}^{d} z_{j} X_{j}\right\}(x),
$$

where $\exp t\left\{\sum_{j} z_{j} X_{j}\right\}, t \in(-\infty, \infty)$ is the one parameter group of diffeomorpshisms generated by the vector field $\sum_{j} z_{j} X_{j}$. Then for each $x$ there exists a neighborhood $V_{x}$ of the origin such that $\phi_{x}: V_{x} \rightarrow U_{x}$ is a diffeomorphism. We define a canonical local coordinate system $\left\{\left(\psi_{x}, U_{x}\right), x \in \mathbf{R}^{d}\right\}$ by $\psi_{x}=\phi_{x}^{-1} ; U_{x} \rightarrow V_{x}$. Then $\psi_{x}(x)=0$ holds for any $x$. Now the solution $\varphi_{t}(x)$ of equation (2) is represented by

$$
\varphi_{t}(x)=\exp \left\{\sum_{j=1}^{d}\left(W^{j}(t)+c^{j} t\right) X_{j}\right\}(x) .
$$

([1], p.210) Then $\psi_{x}\left(\varphi_{t}(x)\right)$ is equal to $\left(W^{1}(t)+c^{1} t, \ldots, W^{d}(t)+c^{d} t\right)$ in $U_{x}$. Therefore for any $x, t, P_{t}\left(x, E \cap U_{x}\right)$ is a Gaussian measure.

If $X_{1}, \ldots, X_{d}$ are not commutative, the process $\psi_{x}\left(\varphi_{t}(x)\right)$ is not a Brownian motion. We will change the scale of the space and time, and consider the process $\varphi_{t}^{(r)}(x)$ represented with the canonical local coordinate $\left(\psi_{x}, U_{x}\right)$ as

$$
\psi_{x}\left(\varphi_{t}^{(r)}(x)\right)=\frac{1}{\sqrt{r}} \psi_{x}\left(\varphi_{r t \wedge \sigma_{x}}(x)\right), \quad t \geq 0,
$$

where $\sigma_{x}=\inf \left\{t>0 ; \varphi_{t} \notin U_{x}\right\}$. Then it converges weakly to a continuous stochastic process $\varphi_{t}^{(0)}(x), t \geq 0$ as $r \rightarrow 0$, which is represented by

$$
\varphi_{t}^{(0)}(x)=\exp \left\{\sum_{j=1}^{d} W^{j}(t) X_{j}\right\}(x) .
$$

Then $\psi_{x}\left(\varphi_{t}^{(0)}(x)\right)$ is equal to $\left(W^{1}(t), \ldots, W^{d}(t)\right)$ in $U_{x}$, proving that for any $x, t$, $P_{t}^{(0)}(x, E):=P\left(\varphi_{t}^{(0)}(x) \in E\right)$ is a Gaussian measure if $E$ is restricted to a Borel subset of $U_{x}$. 
The fact enables us to determine the short time asymptotics of the fundamental solution associated with the operator $(1)$. Let $p_{t}(x, y)$ and $p_{t}^{(0)}(x, y)$ be the density functions of the distributions $P_{t}(x, E)$ and $P_{t}^{(0)}(x, E)$. The former is the fundamental solution associated with the operator (1). The latter is represented by

$$
p_{t}^{(0)}(x, y)=\frac{1}{(2 \pi t)^{d / 2}} \exp \left(-\frac{\left|\psi_{x}(y)\right|^{2}}{2 t}\right) \rho_{x}(y), \quad y \in U_{x}
$$

where

$$
\rho_{x}(y)=\left|\operatorname{det} \frac{\partial \phi_{x}}{\partial z} \circ \psi_{x}(y)\right|^{-1}
$$

Further, we showed in [3] that $p_{t}(x, y) \sim p_{t}^{(0)}(x, y)$ as $t \rightarrow 0$, provided that $y \in U_{x}$.

We shall discuss how the above argument will be extended to the case where $L$ is degenerate. Let $J=\left\{j_{1}, \ldots, j_{l}\right\}$ be a multi-index of $j_{k} \in\{0, \ldots, r\}, k=$ $1, \ldots, l$. We define $X_{J}=\left[X_{j_{1}},\left[X_{j_{2}},\left[\cdots\left[X_{j_{l-1}}, X_{j_{l}}\right] \cdots\right]\right.\right.$. Set $V_{i}=\left\{X_{J} ;\|J\|=\right.$ $i / 2\}$ for $i \geq 1$, where $\|J\|=\sharp\{j \in J ; j=0\}+2^{-1} \sharp\{j \in J ; 1 \leq j \leq r\}$. Then it holds $\mathcal{L} \equiv \cup_{i=1}^{k} V_{i}$ for some $k$. We will choose a basis $\left\{X_{1}, \ldots, X_{n}\right\}$ of $\mathcal{L}$ such that $V_{1}=\left\{X_{1}, \ldots, X_{r_{1}}\right\}, V_{2}-V_{1} \cap V_{2}=\left\{X_{r_{1}+1}, \ldots, X_{r_{2}}\right\}, \cdots, V_{k}-\left(\cup_{j=1}^{k-1} V_{j}\right) \cap$ $V_{k}=\left\{X_{r_{k-1}+1}, \ldots, X_{r_{k}}\right\}$. Associated with the first $d$ vector fields $\left\{X_{1}, \ldots, X_{d}\right\}$ in $\left\{X_{1}, \ldots, X_{n}\right\}$, we define a system of maps $\phi_{x}$ by (3) and introduce a canonical local coordinate system $\left(\psi_{x}, U_{x}\right)$, similarly as the above. Now for $j=1, \ldots, d$, set $\alpha_{j}=2^{-1} l$ if $X_{j} \in V_{l}-\cup_{i=1}^{l-1} V_{i}$. Let $R$ be the $d \times d$ diagonal matrix with diagonal elements $\alpha_{j}, j=1, \ldots, d$. For $r>0$ we set $r^{R}=\exp (\log r) R$. For each $x$, we introduce a family of local diffeomorphisms $\left\{\gamma_{r}^{(x)}, r>0\right\}$ by $\gamma_{r}^{(x)}(y)=\left(\phi_{x} \circ r^{R} \psi_{x}\right)(y), \quad y \in U_{x}$. The point $x$ is invariant by these local diffeomorphisms. It has the group property $\gamma_{r}^{(x)} \circ \gamma_{s}^{(x)}=\gamma_{r s}^{(x)}$ for $r, s>0$. Further it holds $\gamma_{r}^{(x)}(y) \rightarrow x$ as $r \rightarrow 0$ for any $y \in U_{x}$ and $\gamma_{r}^{(x)}(y) \rightarrow \infty$ as $r \rightarrow \infty$ for any $y \in U_{x}$ such that $y \neq x$. We call $\left\{\gamma_{r}^{(x)}\right\}$ a dilation with invariant point $x$.

For $r>0$, we define a stochastic process $\varphi_{t}^{(r)}(x), t \geq 0$ by

$$
\varphi_{t}^{(r)}(x)=\gamma_{1 / r}^{(x)}\left(\varphi_{r t \wedge \sigma_{x}}(x)\right)
$$

where $\sigma_{x}=\inf \left\{t>0, \varphi_{t}(x) \notin U_{x}\right\}$.

Lemma 1 ([3]) The family of stochastic processes $\left\{\varphi_{t}^{(r)}(x), t \geq 0\right\}$ converges weakly as $r \rightarrow 0$. The limit process is equivalent in law with the followings:

$$
\varphi_{t}^{(0)}(x)=\exp \eta_{t}^{(0)}(x), \quad t \geq 0
$$

Here

$$
\eta_{t}^{(0)}=\sum_{j=1}^{d} c_{t}^{j} X_{j}, \quad t \geq 0
$$


where $c_{t}^{j}$ is a linear sum of multiple Wiener-Startonovich integrals

$$
W_{t}^{J}=\int_{0}^{t} \cdots \int_{0}^{t_{2}} \circ d W^{j_{1}}\left(t_{1}\right) \cdots \circ d W^{j_{l}}\left(t_{l}\right),
$$

whose multi-indices $J$ satisfies $\|J\|=\alpha_{j}$ and $P_{X_{j}} X^{J} \neq 0$.

The stochastic process $\left\{\eta_{t}^{(0)}, t \geq 0\right\}$ of $(11)$ is no longer a Brownian motion, provided that $\alpha_{j} \neq 2^{-1}$ for some $1 \leq j \leq d$. However, it is self-similar with respect to $\left\{r^{R}, r>0\right\}$, i.e., the law of the process $\left\{\eta_{r t}^{(0)}, t \geq 0\right\}$ is identical with the law of the process $\left\{r^{R} \eta_{t}^{(0)}, t \geq 0\right\}$ for any $r>0$. Let $F_{t}, t>0$ be distributions of the random variables $\bar{\eta}_{t}^{(0)}, t>0$. They have the self-similar property: $F_{r t}(A)=F_{t}\left(r^{-R} A\right)$ for any $r, t, A$.

Now we introduce Hormander's condition. Let $\mathcal{V}\left(X_{1}, \ldots, X_{r}\right)$ be the smallest class of vector fields such that $X_{i} \in \mathcal{V}\left(X_{1}, \ldots, X_{r}\right)$ for any $i=1, \ldots, r$, and $\left[X, X_{0}\right] \in \mathcal{V}\left(X_{1}, \ldots, X_{r}\right)$ if $X \in \mathcal{V}\left(X_{1}, \ldots, X_{r}\right)$. We introduce

Condition (H). $\operatorname{dim} \operatorname{span}\left\{X(x) ; X \in \mathcal{V}\left(X_{1}, \ldots, X_{r}\right)\right\}=d$ for any $x$ of $\mathbf{R}^{d}$.

Under Condition $(\mathrm{H})$, distributions $F_{t}, t>0$ have $C^{\infty}$ density functions $f_{t}(z)$. They satisfy

$$
f_{r t}(z)=\frac{1}{r^{\operatorname{tr} R}} f_{t}\left(r^{-R} z\right)
$$

because of the self-similarity of distributions $F_{t}$.

Theorem 2 ([3]) Assume Condition (H). Then the distribution $P_{t}^{(0)}(x, E)$ of $\varphi_{t}^{(0)}(x)$ has a $C^{\infty}$-density function $p_{t}^{(0)}(x, y)$. Further, it is represented by

$$
p_{t}^{(0)}(x, y)=\frac{1}{t^{\operatorname{tr} R}} f_{1}\left(t^{-R} \psi_{x}(y)\right) \rho_{x}(y), \quad \forall y \in U_{x}
$$

where $\rho_{x}(y)$ is given by (8).

Theorem 3 ([3]) Let $p_{t}(x, y)$ be the fundamental solution associated with the operator $L$ of (1). Assume Condition (H). Then, for any $y \in U_{x}$, it holds

$$
p_{t}(x, y) \sim p_{t}^{(0)}(x, y) \quad \text { as } t \rightarrow 0
$$

\section{SPDE AND ASSOCIATED RANDOM TRANSITION FUNCTIONS}

We shall apply the above argument to the study of the short time asymptotics of the random fundamental solution associated with the stochastic partial differential operator

$$
A(t)=\left(\frac{1}{2} \sum_{j=1}^{r} X_{j}^{2}+X_{0}\right) t+\sum_{k=1}^{m} Y_{k} W^{k}(t)
$$


where $Y_{1}, \ldots, Y_{m}$ are linearly independent complete $C^{\infty}$ vector fields on $\mathbf{R}^{d}$ and $\left(W^{1}(t), \ldots, W^{m}(t)\right)$ is a standard $m$-dimensional Brownian motion on the probability space $(\Omega, \mathcal{F}, P)$. We assume that $\left\{X_{0}, X_{1}, \ldots, X_{r}, Y_{1}, \ldots, Y_{m}\right\}$ generates a finite dimensional Lie algebra $\tilde{\mathcal{L}}$.

We shall first construct random transition probabilities associated with the random infinitesimal generator $A(t)$ of (16). Let $\mathbf{C}=C\left([0, \infty), \mathbf{R}^{r}\right)$ be the spaces of continuous paths starting from the origin of the Euclidean spaces $\mathbf{R}^{r}$. Elements of $\mathbf{C}$ are denoted by $w$ and their values at $t$ are denoted by $B(t)$, i.e., $B(t)(w)=w(t)$. Let $Q$ be a Wiener measure on C. We consider the product probability space $(\Omega \times \mathbf{C}, P \otimes Q)$. Then $\left\{B(t)=\left(B^{1}(t), \ldots, B^{r}(t)\right), t \geq 0\right\}$ and $\left\{W(t)=\left(W^{1}(t), \ldots, W^{m}(t)\right), t \geq 0\right\}$ are independent standard Brownian motions on the product probability space.

Consider a stochastic differential equation on the probability space $P \times Q$.

$$
\begin{aligned}
d \xi_{t} & =\sum_{j=1}^{r} X_{j}\left(\xi_{t}\right) \circ d B^{j}(t)+X_{0}\left(\xi_{t}\right) d t+\sum_{k=1}^{m} Y_{k}\left(\xi_{t}\right) \circ d W^{k}(t), \quad t>s \\
\xi_{s} & =x, \quad x \in \mathbf{R}^{d} .
\end{aligned}
$$

The solution $\xi_{s, t}(x)=\xi_{s, t}(x, \omega, w), t \geq s$ of the above equation defines a stochastic flow of $C^{\infty}$-diffeomorphisms of $\mathbf{R}^{d}$. We define random transition probabilities $\tilde{P}_{s, t}(x, E)$ by

$$
\tilde{P}_{s, t}(x, E, \omega)=Q\left(\xi_{s, t}(x, \omega, \cdot) \in E\right) .
$$

and the random positive semigroup by

$$
\tilde{T}_{s, t} f(x)=\int_{\mathbf{R}^{d}} \tilde{P}_{s, t}(x, d y) f(y) .
$$

Its random infinitesimal generator (in Stratonovich sense) is given by (17), that is to say, it satisfies

$$
\tilde{T}_{s, t} f=f+\int_{s}^{t} \tilde{T}_{s, r} A(\circ d r) f
$$

for any $C^{\infty}$ function $f$ with bounded derivatives.

Now let $\mathcal{V}\left(X_{1}, \ldots, X_{r}\right)$ be the smallest class of vector fields such that (1) $X_{i} \in \mathcal{V}\left(X_{1}, \ldots, X_{r}\right)$ for any $i=1, \ldots, r,(2)$ If $X \in \mathcal{V}\left(X_{1}, \ldots, X_{r}\right)$ then $\left[Y_{j}, X\right] \in$ $\mathcal{V}\left(X_{1}, \ldots, X_{r}\right)$ for all $j=0,1, \ldots, m$. We introduce a condition similar to Hörmander's condition $(\mathrm{H})$.

Condition $(\tilde{H}) . \operatorname{dim} \operatorname{span}\left\{X(x) ; X \in \mathcal{V}\left(X_{1}, \ldots, X_{r}\right)\right\}=d$ for any $x$ of $\mathbf{R}^{d}$. Then it is shown in [2] that there exists a random function $\tilde{p}_{s, t}(x, y)=\tilde{p}_{s, t}(x, y, \omega)$, $C^{\infty}$ with respect to $x, y$, such that

$$
\tilde{P}_{s, t}(x, d y)=\tilde{p}_{s, t}(x, y) d y, \quad \text { a.s. } P .
$$

We call $\tilde{p}_{s, t}(x, y)$ the random fundamental solution or random heat kernel associated with the operator $A(t)$. It satisfies the following properties. 
(1) $\tilde{p}_{s, t}(x, y)$ is nonnegative and continuous in $(s, x, t, y)$ a.s. $P$. Further, it is a $\mathbf{C}^{\infty}$-function of $x$ and $y$ a.s. $P$

(2) For any $s<t<u$,

$$
\tilde{p}_{s, u}(x, z)=\int \tilde{p}_{s, t}(x, y) \tilde{p}_{t, u}(y, z) d y, \quad \forall x, z \in \mathbf{R} \quad \text { a.s. } P .
$$

(3) Kolmogorov's backward equation holds true:

$$
\tilde{p}_{s, t}(x, y)=\delta_{t, y}+\int_{s}^{t} A_{x}(\circ \hat{d} r) \tilde{p}_{r, t}(x, y), \quad \forall s<t, \forall x, y \in \mathbf{R}^{d} \quad \text { a.s. } \quad P \text {. }
$$

(4) Kolmogorov's forward equation holds true:

$$
\tilde{p}_{s, t}(x, y)=\delta_{s, x}+\int_{s}^{t} A_{y}^{\prime}(\circ d r) \tilde{p}_{s, r}(x, y), \quad \forall s<t, \forall x, y \in \mathbf{R}^{d} \quad \text { a.s. } \quad P .
$$

Here $A^{\prime}(t)$ is the formal adjoint of $A(t)$, i.e., $\int A(t) f g d x=\int f A^{\prime}(t) g d x$ holds a.s. for any $C^{\infty}$-functions $f, g$ with compact supports.

\section{ASYMPTOTICS OF RANDOM TRANSITION PROBABILITIES}

We are interested in the asymptotics of random transition probabilities $\tilde{P}_{s, t}(x, E)$ and random fundamental solutions $\tilde{p}_{s, t}(x, y)$ as $t$ tends to $s$. For this purpose we shall introduce a system of canonical local coordinates associated with the random differential operator $A(t)$.

For the convenience of notations, we set $X_{r+1}=X_{0}, X_{r+2}=Y_{1}, \ldots, X_{r+m+1}=$ $Y_{m}$. Let $J=\left\{j_{1}, \ldots, j_{l}\right\}$ be a multi-index of $j_{i} \in\{1, \ldots, r+m+1\}$. Define $\tilde{V}_{i}=\left\{X_{J} ;\langle J\rangle=i\right\}, i=1, \ldots$, where $\langle J\rangle=\sharp\left\{j \in \tilde{\mathcal{C}}_{\tilde{X}} J 1 \leq j \leq r\right\}$ It holds $\tilde{\mathcal{L}}=\cup_{i=1}^{k} \tilde{V}_{i}$ for some $k<\infty$. We will choose a basis $\left\{\tilde{X}_{1}, \ldots, \tilde{\tilde{X}}_{n}\right\}$ of $\tilde{\mathcal{L}}$ such that $\tilde{V}_{1}=\left\{\tilde{\tilde{X}}_{1}, \ldots, \tilde{X}_{r_{1}}\right\}, \tilde{V}_{i}-\left(\cup_{j=1}^{i-1} \tilde{V}_{j}\right) \cap \tilde{V}_{i}=\left\{\tilde{X}_{r_{i-1}+1}, \ldots, \tilde{X}_{r_{i}}\right\}$. Associated with the first $d$ vector fields $\left\{\tilde{X}_{1}, \ldots, \tilde{X}_{d}\right\}$ in $\tilde{\mathcal{L}}$, we define a system of maps $\tilde{\phi}_{x} ; \mathbf{R}^{d} \rightarrow \mathbf{R}^{d}$ with parameter $x$ by $\tilde{\phi}_{x}(z)=\exp \sum_{j=1}^{d} z_{j} \tilde{X}_{j}(x)$. Then the canonical local coordinate system $\left\{\left(\tilde{\psi}_{x}, \tilde{U}_{x}\right), x \in \mathbf{R}^{d}\right\}$ is introduced similarly as before.

Let $\tilde{R}$ be a $d \times d$ diagonal matrix, whose diagonal elements are $\tilde{\alpha}_{j}=2^{-1} i$, $j=1, \ldots, d$ if $\tilde{X}_{j} \in \tilde{V}_{i}-\cup_{j \leq i-1} \tilde{V}_{j}$. We define a dilation $\left\{\tilde{\gamma}_{r}^{(x)}\right\}$ for each $x$ as $\tilde{\gamma}_{r}^{(x)}(y)=\left(\tilde{\phi}_{x} \circ r^{\tilde{R}} \tilde{\psi}_{x}\right)(y), \quad y \in \tilde{U}_{x}$.

We shall rewrite equation (17) using new vector fields $\tilde{X}_{1}, \cdots, \tilde{X}_{n}$. Denote the projection of $X_{k}$ (or $Y_{k}$ ) to the space $\left\{\tilde{X}_{j}\right\}$ by $c_{j k} \tilde{X}_{j}$ (or $d_{j k} \tilde{X}_{j}$ ) and set $\tilde{W}_{j}(t)=\sum_{k} d_{j k} W^{k}(t)$ (or $\tilde{B}_{j}(t)=\sum_{k} c_{j k} B^{k}(t)$ ). Then equation (17) is written as

$$
d \xi_{t}=\sum_{j=1}^{n} \tilde{X}_{j}\left(\xi_{t}\right) \circ d Z^{j}(t), \quad \text { where } Z^{j}(t)=\tilde{B}^{j}(t)+\tilde{W}^{j}(t)
$$

Define

$$
\xi_{s, s+t}^{(r)}(x)=\tilde{\gamma}_{1 / r}^{(x)}\left(\xi_{s,(s+r t) \wedge \tilde{\sigma}_{x}}(x)\right)
$$


where $\tilde{\sigma}_{x}=\inf \left\{t>s, \xi_{s, t}(x) \notin \tilde{U}_{x}\right\}$.

Lemma 4 Let $r \rightarrow 0$. Then the family of stochastic processes $\left\{\xi_{s, s+t}^{(r)}(x), t \geq\right.$ $0\}, r>0$ converges weakly to $\left\{\xi_{s, s+t}^{(0)}(x), t \geq 0\right\}$. Further, it is represented by

$$
\xi_{s, s+t}^{(0)}(x)=\exp \eta_{s, s+t}^{(0)}(x) .
$$

Here

$$
\eta_{s, s+t}^{(0)}=\sum_{j=1}^{d} c_{s, s+t}^{j} \tilde{X}_{j}
$$

where $c_{s, s+t}^{j}$ is a linear sum of multiple Wiener-Stratonovich integrals

$$
Z_{s, s+t}^{J}=\int_{s}^{s+t} \cdots \int_{s}^{s+t_{2}} d Z^{j_{1}}\left(t_{1}\right) \cdots \circ d Z^{j_{l}}\left(t_{l}\right),
$$

whose multi-indecis $J$ satisfies $\|J\|=\tilde{\alpha}_{j}$ and $P_{\tilde{X}_{j}} \tilde{X}_{J} \neq 0$.

The stochastic process $\eta_{s, s+t}^{(0)}$ is self-similar with respect to $r^{\tilde{R}}$, i.e., the law of $\left(\eta_{s, s+r t}^{(0)}, P \times Q\right)$ is identical with that of $\left(r^{\tilde{R}} \eta_{s, s+t}^{(0)}, P \times Q\right)$ for any $s, t, r$. Let $F_{s, t}=F_{s, t}(\omega)$ be the conditional distribution given $\omega$, i.e., $F_{s, t}(A, \omega)=$ $Q\left(\eta_{s, t}^{(0)}(\omega, \cdot) \in A\right)$. It has the self-similar property $F_{s, s+r t}(A, \omega)=F_{s, s+t}\left(r^{-\tilde{R}} A, \omega^{(r)}\right)$ holds for any $s, t, r, A$, where $\omega^{(r)}$ is the sample such that $W_{t}\left(\omega^{(r)}\right)=W_{r t}(\omega)$ holds for all $t>0$. Assume Condition $(\tilde{H})$. Then $F_{s, t}$ has a $C^{\infty}$ density function $f_{s, t}(z)=f_{s, t}(z, \omega)$. It satisfies

$$
f_{s, s+r t}(z, \omega)=\frac{1}{r^{\operatorname{tr} \tilde{R}}} f_{s, s+t}\left(r^{-\tilde{R}} z, \omega^{(r)}\right), \quad \text { a.s. } P,
$$

for any $r>0$.

We can rewrite $\eta_{s, t}^{(0)}$ of $(28)$ as: $\eta_{s, t}^{(0)}=\sum_{1 \leq j \leq d}\left(d_{s, t}^{j}+e_{s, t}^{j}\right) \tilde{X}_{j}$. Here, $d_{s, t}^{j}$ is the collection of terms $Z^{J}(t)$ in $c_{s, t}^{j}$ such that $\langle J\rangle>0$, and $e_{s, t}^{j}$ is the collection of terms in $c_{s, t}^{j}$ such that $\langle J\rangle=0$. We define $\hat{\eta}_{s, t}^{(0)}=\sum_{1 \leq j \leq d} d_{s, t}^{j} X_{j}$. Let $\hat{F}_{s, t}(A, \omega)=Q\left(\hat{\eta}_{s, t}^{(0)}(\omega, \cdot) \in A\right)$. Under Condition $(\tilde{H}), \hat{F}_{s, t}$ has also a $C^{\infty}$ density function $\hat{f}_{s, t}(z)=\hat{f}_{s, t}(z, \omega)$. It satisfies the equality similar to $(30)$. We have further,

$$
f_{s, t}(z)=\hat{f}_{s, t}\left(z-e_{s, t}\right) .
$$

Theorem 5 Assume Condition $(\tilde{H})$. Let $\tilde{P}_{s, t}^{(0)}(x, E, \omega)=Q\left(\xi_{s, t}^{(0)}(x, \omega, \cdot) \in\right.$ $E)$. Then $\tilde{P}_{s, t}^{(0)}(x, \cdot, \omega)$ has a $C^{\infty}$ density function $\tilde{p}_{s, t}^{(0)}(x, y, \omega)$ a.s. P. Further, it is represented by

$$
\begin{aligned}
\tilde{p}_{s, s+t}^{(0)}(x, y, \omega) & =f_{s, s+t}\left(\tilde{\psi}_{x}(y), \omega\right) \tilde{\rho}_{x}(y), \quad \text { a.s. } P, \\
& =\frac{1}{t^{\operatorname{tr} \tilde{R}}} \hat{f}_{s, s+1}\left(t^{-\tilde{R}}\left(\tilde{\psi}_{x}(y)-e_{s, t}\right), \omega^{(t)}\right) \tilde{\rho}_{x}(y), \quad \text { a.s. } P
\end{aligned}
$$


if $y \in U_{x}$, where $\tilde{\rho}(x)$ is defined by (8) replacing $\phi_{x}$ and $\psi_{x}$ by $\tilde{\phi}_{x}$ and $\tilde{\psi}_{x}$, respectively.

Finally, we consider the case where the operator $A(t)$ of $(16)$ is nondegenerate. The diagonal matrix $\tilde{R}$ coincides with $2^{-1} I$, where $I$ is the identity matrix. Further, in the expression of $\eta_{s . s+t}^{(0)}$, multiple Wiener-Stratonovich integrals are not involved so that $\eta_{s, s+t}^{(0)}$ is a linear sum of Brownian motions $Z^{1}(t), \ldots, Z^{m}(t)$. Then the conditional distribution of $\eta_{s, s+t}^{(0)}$ with respect to $Q$ is Gaussian. Indeed, we have

Corollary 6 Suppose that $A(t)$ is nondegenerate. Then, if $y \in U_{x}$,

$\tilde{p}_{s, s+t}^{(0)}(x, y)=\frac{1}{(2 \pi t)^{d / 2}} \exp \left(-\frac{\left|\tilde{\psi}_{x}(y)-(W(s+t)-W(s))\right|^{2}}{2 t}\right) \tilde{\rho}_{x}(y), \quad$ a.s. $P$.

Theorem 7 Assume Condition $(\tilde{H})$. Let $\tilde{p}_{s, s+t}(x, y)$ be the fundamental solution associated with the random differential operator $A(t)$. Then we have for $y \in U_{x}$,

$$
\tilde{p}_{s, s+t}(x, y) \sim \tilde{p}_{s, s+t}^{(0)}(x, y) \quad \text { as } t \rightarrow 0 \quad \text { a.s. } P .
$$

The proof of theorems in this section will be given elsewhere.

\section{References}

[1] H. Kunita, Stochastic flows and stochastic differential equations, Cambridge Univ. Press, 1990.

[2] H. Kunita, Generalized solutions of stochastic partial differential equations, J. Theoretical Probability 7(1994), 279-308.

[3] H. Kunita, Asymptotic self-similarity and short time asympttics of stochastic flows, J. Univ. Tokyo

[4] S. Kusuoka, D.W. Stroock, Application of the Malliavin calculus, Part II, J. Fac. Sci. Univ. Tokyo 32(1985), 1-76.

[5] S. Kusuoka, D.W. Stroock, Application of the Malliavin calculus, Part III, J. Fac. Sci. Univ. Tokyo 32(1985), 103-147.

[6] S. Takanobu, Diagonal short time asymptotics fo Heat kernels for certain degenerate second order differential operators of Hörmander type, Pub. Res. Inst. Math. Sci. 24(1988), 169-203. 